\title{
THE TORSION AND STRETCHING OF SPIRAL RODS (I)*
}

\author{
BY H. OKKUBO \\ (Institute of High Speed Mechanics, \\ Tōhoku University, Sendai, Japan)
}

\section{SYNOPSIS}

In this paper the torsion or the stretching problem for a spiral rod is treated theoretically. The equations of equilibrium expressed in terms of displacements are reduced to forms which are independent of one co-ordinate. They are readily integrated for the particular case where the helix angle is small, and the corresponding displacements and stresses can be expressed in forms which contain three arbitrary plane harmonic functions, determination of which is dependent upon the shape of the section. As an application of the general solution, the problem for an elliptic section is solved explicitly.

Two-dimensional problems in elasticity have been studied extensively from early times on account of their simplicity in stress analysis and their useful applications in many engineering problems. For a similar reason, various problems of axially symmetrical stress distribution have been investigated by many writers.

In this paper we shall treat the torsion or the stretching problem for a spiral rod. The stress distribution for this case is neither two-dimensional nor axially symmetrical, and each stress does not vanish in general and consequently the analysis becomes somewhat complicated. But the problem is not a three dimensional one without any restriction, since if we rotate the co-ordinate axes about the axis of the helix so as to coincide with the fixed directions with respect to a section which is perpendicular to the axis of helix, then the stress distribution referred to the rotating axes is the same in any section.

Starting from the equations of equilibrium expressed in terms of displacements, we shall introduce equations which are independent of the position of the section. The differential equations of displacements are readily integrated for the particular case where the helix angle is small. The corresponding displacements and stresses are expressed in forms which contain three arbitrary plane harmonic functions, determination of which is dependent upon the shape of the section, and thus we can considerably simplify the problem.

We shall take the axis of the helix as the $z$-axis, and shall denote the displacements in the $x, y, z$ directions by $u, v$ and $w$, respectively. Then the equations of equilibrium can be expressed in the forms ${ }^{1}$

$$
\begin{aligned}
& (\lambda+\mu) \frac{\partial \Delta}{\partial x}+\mu \nabla^{2} u=0, \\
& (\lambda+\mu) \frac{\partial \Delta}{\partial y}+\mu \nabla^{2} v=0, \\
& (\lambda+\mu) \frac{\partial \Delta}{\partial z}+\mu \nabla^{2} w=0,
\end{aligned}
$$

where $\lambda, \mu$ are Lamé constants and $\Delta$ is the cubical dilatation.

*Received November 16, 1950.

${ }^{1} \mathrm{H}$. Love, The mathematical.theory of elasticity, 4th ed., 1927, p. 133. 
We shall make the following transformation

$$
x^{\prime}+i y^{\prime}=e^{i k z}(x+i y),
$$

where $k$ is a constant which is related to the obliquity of the helix.

Let $u^{\prime}, v^{\prime}$ be the displacements in the $x^{\prime}$ and $y^{\prime}$ directions respectively. It follows that

$$
u^{\prime}+i v^{\prime}=e^{i k z}(u+i v) \text {. }
$$

Let us put the displacements in the form

$$
\begin{aligned}
& u^{\prime}=u_{1}\left(x^{\prime}, y^{\prime}\right)-\alpha y^{\prime} z, \\
& v^{\prime}=v_{1}\left(x^{\prime}, y^{\prime}\right)+\alpha x^{\prime} z, \\
& w=w_{1}\left(x^{\prime}, y^{\prime}\right)+\beta z,
\end{aligned}
$$

where $\alpha, \beta$ are constants. Using the expressions for the displacements in Eq. (4), we obtain the cubical dilatation

$$
\Delta=\frac{\partial u}{\partial x}+\frac{\partial v}{\partial y}+\frac{\partial w}{\partial z}=\frac{\partial u_{1}}{\partial x^{\prime}}+\frac{\partial v_{1}}{\partial y^{\prime}}-k D_{2}\left(w_{1}\right)+\beta,
$$

and

$$
\frac{\partial \Delta}{\partial x}=\frac{\partial \Delta}{\partial x^{\prime}} \cos k z+\frac{\partial \Delta}{\partial y^{\prime}} \sin k z .
$$

Remembering the relation in Eq. (3), we have

$$
\begin{aligned}
\nabla^{2} u=\left\{\nabla_{1}^{2} u_{1}\right. & \left.+k^{2} D_{1}\left(u_{1}\right)-2 k^{2} D_{2}\left(v_{1}\right)\right\} \cos k z \\
& +\left\{\nabla_{1}^{2} v_{1}+k^{2} D_{1}\left(v_{1}\right)+2 k^{2} D_{2}\left(u_{1}\right)\right\} \sin k z,
\end{aligned}
$$

where the operators $D_{1}, D_{2}$ and $\nabla_{1}$ are

$$
\begin{aligned}
& D_{1}=y^{\prime 2} \frac{\partial^{2}}{\partial x^{\prime 2}}-2 x^{\prime} y^{\prime} \frac{\partial^{2}}{\partial x^{\prime} \partial y^{\prime}}+x^{\prime 2} \frac{\partial^{2}}{\partial y^{\prime 2}}-x^{\prime} \frac{\partial}{\partial x^{\prime}}-y^{\prime} \frac{\partial}{\partial y^{\prime}}-1, \\
& D_{2}=y^{\prime} \frac{\partial}{\partial x^{\prime}}-x^{\prime} \frac{\partial}{\partial y^{\prime}}, \quad \nabla_{1}^{2}=\frac{\partial^{2}}{\partial x^{\prime 2}}+\frac{\partial^{2}}{\partial y^{\prime 2}} .
\end{aligned}
$$

To satisfy the first equation in (1), we have

$$
\begin{aligned}
& (\lambda+\mu) \frac{\partial \Delta}{\partial x^{\prime}}+\mu\left\{\nabla_{1}^{2} u_{1}+k^{2} D_{1}\left(u_{1}\right)-2 k^{2} D_{2}\left(v_{1}\right)\right\}=0, \\
& (\lambda+\mu) \frac{\partial \Delta}{\partial y^{\prime}}+\mu\left\{\nabla_{1}^{2} v_{1}+k^{2} D_{1}\left(v_{1}\right)+2 k^{2} D_{2}\left(u_{1}\right)\right\}=0 .
\end{aligned}
$$

The displacements which satisfy the above conditions, also satisfy the second equation in (1). From the third equation in (1) we have the relation

$$
-k(\lambda+\mu) D_{2}(\Delta)+\mu\left\{\nabla_{1}^{2} w_{1}+k^{2} D_{1}\left(w_{1}\right)+k^{2} w_{1}\right\}=0 .
$$


Accordingly, Eqs. (5) and (6) are the equations of equilibrium for this case. Integrating these differential equations, we obtain $u_{1}, v_{1}$ and $w_{1}$, which are independent of $z$. Substituting them in the following equations

$$
\begin{aligned}
& X_{x^{\prime}}^{\prime}=\lambda \Delta+2 \mu \frac{\partial u_{1}}{\partial x^{\prime}}, \\
& Y_{y^{\prime}}^{\prime}=\lambda \Delta+2 \mu \frac{\partial v_{1}}{\partial y^{\prime}}, \\
& Z_{z}=\lambda \Delta+2 \mu\left(\beta-k D_{2}\left(w_{1}\right)\right), \\
& X_{y^{\prime}}^{\prime}=\mu\left(\frac{\partial u_{1}}{\partial y^{\prime}}+\frac{\partial v_{1}}{\partial x^{\prime}}\right), \\
& X_{z}^{\prime}=\mu\left(\frac{\partial w_{1}}{\partial x^{\prime}}-k D_{2}\left(u_{1}\right)+k v_{1}-\alpha y^{\prime}\right), \\
& Y_{z}^{\prime}=\mu\left(\frac{\partial w_{1}}{\partial y^{\prime}}-k D_{2}\left(v_{1}\right)-k u_{1}+\alpha x^{\prime}\right),
\end{aligned}
$$

we obtain the stresses, which are independent of $z$.

If we express the equation representing the bounding curve of the section by

$$
F\left(x^{\prime}, y^{\prime}\right)=0 \text {, }
$$

the condition that the bounding surface of the rod is free from traction is satisfied if the equations

$$
\begin{aligned}
& X_{x^{\prime}}^{\prime} \frac{\partial F}{\partial x^{\prime}}+X_{\nu^{\prime}}^{\prime} \frac{\partial F}{\partial y^{\prime}}-k D_{2}\left(F^{\prime}\right) X_{z}^{\prime}=0 \\
& X_{v^{\prime}}^{\prime} \frac{\partial F}{\partial x^{\prime}}+Y_{\nu^{\prime}}^{\prime} \frac{\partial F}{\partial y^{\prime}}-k D_{2}\left(F^{\prime}\right) Y_{z}^{\prime}=0 \\
& X_{z}^{\prime} \frac{\partial F}{\partial x^{\prime}}+Y_{z}^{\prime} \frac{\partial F}{\partial y^{\prime}}-k D_{2}(F) Z_{z}=0
\end{aligned}
$$

hold at all points of the bounding curve of the section.

Let us consider the equilibrium of a portion of the rod cut by two parallel planes perpendicular to the axis of the helix. Since the stresses in Eq. (7) are independent of $z$, the resultant of the shearing stress on each plane is the same in magnitude but generally different in direction; and from the condition of equilibrium of tractions, the resultant of the shearing stress vanishes. The effect of the normal traction on each plane is statically equivalent to a single force and a couple. The former can be cancelled by taking the constant $\beta$ so as to satisfy the condition

$$
\iint Z_{z} d x^{\prime} d y^{\prime}=0
$$

and by a similar consideration, we see that the latter vanishes. Accordingly, the effect 
of all tractions acting on each plane is equivalent to a couple due to the shearing force, and the solution which satisfies Eqs. (5) and (6) with the boundary conditions (9) and (10) is the one for the torsion problem of the rod. If we determine $\alpha$ so as to satisfy the condition that the moment of the couple due to the shearing force vanishes on each plane, instead of employing condition (10), then we have a solution for the problem of the stretching of a rod.

First, we shall consider the torsion problem. When $k$ is small, $u_{1}, v_{1}$ and $\Delta$ are small quantities since they all vanish for a straight rod, and so if we neglect the smaller quantities of the second order, the equations of equilibrium (5) and (6) can be written in simpler forms as

$$
\begin{aligned}
\frac{\partial \Delta}{\partial x^{\prime}}+\frac{\mu}{\lambda+\mu} \nabla_{1}^{2} u_{1} & =0, \\
\frac{\partial \Delta}{\partial y^{\prime}}+\frac{\mu}{\lambda+\mu} \nabla_{1}^{2} v_{1} & =0, \\
\nabla_{1}^{2} w_{1} & =0,
\end{aligned}
$$

and the third equation of the boundary condition (9) becomes

$$
X_{z}^{\prime} \frac{\partial F}{\partial x^{\prime}}+Y_{z}^{\prime} \frac{\partial F}{\partial y^{\prime}}=0
$$

and the stresses $X_{z}^{\prime}$ and $Y_{z}^{\prime}$ become

$$
X_{z}^{\prime}=\mu\left(\frac{\partial w_{1}}{\partial x^{\prime}}-\alpha y^{\prime}\right), \quad Y_{z}^{\prime}=\mu\left(\frac{\partial w_{1}}{\partial y^{\prime}}+\alpha x^{\prime}\right) .
$$

Accordingly, when $k$ is small, the shearing stresses $X_{z}^{\prime}$ and $Y_{z}^{\prime}$ and hence the torque acting on the rod are the same as those for a straight cylinder, but the other stresses do not vanish as in the latter case.

Let us put

$$
w_{1}=i\left(f_{3}-\bar{f}_{3}\right),
$$

where $f_{3}$ is an arbitrary function and $f_{3}=f_{3}(\zeta), \bar{f}_{3}=f_{3}(\bar{\zeta}), \zeta=x^{\prime}+i y^{\prime}$ and $\bar{\zeta}=x^{\prime}-i y^{\prime}$. $w_{1}$ satisfies the third equation in (11). Substituting this expression for $w_{1}$ into the first and second equations in (11), we have

$$
\begin{aligned}
& \frac{\lambda+2 \mu}{\lambda+\mu} \frac{\partial^{2} u_{1}}{\partial x^{\prime 2}}+\frac{\partial^{2} v_{1}}{\partial x^{\prime} \partial y^{\prime}}+\frac{\mu}{\lambda+\mu} \frac{\partial^{2} u_{1}}{\partial y^{\prime 2}}=k\left[f_{3}^{\prime}+\overline{f_{3}^{\prime}}+\zeta f_{3}^{\prime \prime}+\bar{\zeta} \overline{f_{3}^{\prime \prime}}\right] \\
& \frac{\mu}{\lambda+\mu} \frac{\partial^{2} v_{1}}{\partial x^{\prime 2}}+\frac{\partial^{2} u_{1}}{\partial x^{\prime} \partial y^{\prime}}+\frac{\lambda+2 \mu}{\lambda+\mu} \frac{\partial^{2} v_{1}}{\partial y^{\prime 2}}=i k\left[f_{3}^{\prime}-\overline{f_{3}^{\prime}}+\zeta f_{3}^{\prime \prime}-\overline{\zeta f_{3}^{\prime \prime}}\right]
\end{aligned}
$$

The particular solution of Eq. (15) is

$$
u_{1}=k \int f_{3}^{\prime} \zeta d \zeta+k \int \overline{f_{3}^{\prime}} \bar{\zeta} d \bar{\zeta}, \quad v_{1}=0 .
$$

Setting the right sides of Eqs. (15) equal to zero, we obtain the relations

$$
\nabla_{1}^{4} u_{1}=0, \quad \text { or } \quad \nabla_{1}^{4} v_{1}=0,
$$


from which we can put

$$
u_{1}=f_{1}+\overline{f_{1}}+x^{\prime}\left(f_{2}+\overline{f_{2}}\right),
$$

where $f_{1}$ and $f_{2}$ are arbitrary functions of $\zeta$. Inserting this expression for $u_{1}$ into (15), we have

$$
v_{1}=i\left(f_{1}-\overline{f_{1}}\right)+i x^{\prime}\left(f_{2}-\overline{f_{2}}\right)+i \frac{\lambda+3 \mu}{\lambda+\mu}\left[\int f_{2} d \zeta-\int \overline{f_{2}} d \bar{\zeta}\right] .
$$

Accordingly, the displacements which satisfy the equations of equilibrium (11) can be expressed in the forms

$$
\begin{aligned}
& u^{\prime}=f_{1}+\overline{f_{1}}+x^{\prime}\left(f_{2}+\overline{f_{2}}\right)+k\left[\int f_{3}^{\prime} \zeta d \zeta+\int \overline{f_{3}^{\prime} \zeta} d \bar{\zeta}\right]-\alpha y^{\prime} z \\
& v^{\prime}=i\left(f_{1}-\overline{f_{1}}\right)+i x^{\prime}\left(f_{2}-\overline{f_{2}}\right)+i(2 p+1)\left[\int f_{2} d \zeta-\int \overline{f_{2}} d \bar{\zeta}\right]+\alpha x^{\prime} z \\
& w=i\left(f_{3}-\overline{f_{3}}\right)+\beta z
\end{aligned}
$$

and the corresponding expressions for the stresses are given in the forms

$$
\begin{aligned}
& X_{x^{\prime}}^{\prime}=2 \mu\left\{f_{1}^{\prime}+\overline{f_{1}^{\prime}}+p\left(f_{2}+\overline{f_{2}}\right)+x^{\prime}\left(f_{2}^{\prime}+\overline{f_{2}^{\prime}}\right)+k\left(\zeta f_{3}^{\prime}+\bar{\zeta} \overline{f_{3}^{\prime}}\right)\right\}+\lambda \beta, \\
& Y_{y^{\prime}}^{\prime}=-2 \mu\left\{f_{1}^{\prime}+\overline{f_{1}^{\prime}}+(p+2)\left(f_{2}+\overline{f_{2}}\right)+x^{\prime}\left(f_{2}^{\prime}+\overline{f_{2}^{\prime}}\right)\right\}+\lambda \beta, \\
& Z_{z}=-2 \mu\left\{(1-p)\left(f_{2}+\overline{f_{2}}\right)+k\left(\zeta f_{3}^{\prime}+\bar{\zeta} \overline{f_{3}^{\prime}}\right)\right\}+(\lambda+2 \mu) \beta, \\
& X_{y^{\prime}}^{\prime}=2 i \mu\left\{f_{1}^{\prime}-\overline{f_{1}^{\prime}}+x^{\prime}\left(f_{2}^{\prime}-\overline{f_{2}^{\prime}}\right)+(p+1)\left(f_{2}-\overline{f_{2}}\right)+\frac{k}{2}\left(\zeta f_{3}^{\prime}-\overline{\zeta f_{3}^{\prime}}\right)\right\}, \\
& X_{z}^{\prime}=\mu\left\{i\left(f_{3}^{\prime}-\overline{f_{3}^{\prime}}\right)-\alpha y^{\prime}\right\}, \\
& Y_{z}^{\prime}=-\mu\left\{f_{3}^{\prime}+\overline{f_{3}^{\prime}}-\alpha x^{\prime}\right\},
\end{aligned}
$$

where

$$
p=\frac{\mu}{\lambda+\mu} .
$$

As an example, we shall consider an elliptic spiral rod whose section is given by

$$
\frac{x^{\prime 2}}{a^{2}}+\frac{y^{\prime 2}}{b^{2}}=1 \text {. }
$$

Let us put

$$
2 f_{1}^{\prime}=A_{0}+A_{1} \zeta^{2}, \quad 2 f_{2}=B_{0}+B_{1} \zeta^{2}, \quad 2 f_{3}^{\prime}=h \zeta
$$

where

$$
h=\frac{a^{2}-b^{2}}{a^{2}+b^{2}} \alpha
$$


Substituting the expressions in (20) into (18), we obtain

$$
\begin{aligned}
& X_{x^{\prime}}^{\prime}=2 \mu\left\{A_{0}+p B_{0}+2 B_{1}{x^{\prime}}^{2}+\left(A_{1}+p B_{1}+k h\right)\left({x^{\prime}}^{2}-y^{\prime 2}\right)\right\}+\lambda \beta, \\
& Y_{y^{\prime}}^{\prime}=-2 \mu\left\{A_{0}+(p+2) B_{0}+2 B_{1} x^{\prime 2}+\left[A_{1}+(p+2) B_{1}\right]\left(x^{\prime 2}-y^{\prime 2}\right)\right\}+\lambda \beta, \\
& Z_{z}=-2 \mu\left\{(1-p) B_{0}+\left[(1-p) B_{1}+k h\right]\left(x^{\prime 2}-y^{\prime 2}\right)\right\}+(\lambda+2 \mu) \beta, \\
& X_{y^{\prime}}^{\prime}=-2 \mu\left\{2 A_{1}+2(p+2) B_{1}+k h\right\} x^{\prime} y^{\prime}, \\
& X_{z}^{\prime}=-\mu(h+\alpha) y^{\prime}, \\
& Y_{z}^{\prime}=-\mu(h-\alpha) x^{\prime} .
\end{aligned}
$$

From the boundary conditions (9) and (10) we obtain a system of simultaneous equations for the determination of the unknown constants $A_{n}, B_{0}, A_{1}, B_{1}$ and $\beta$ as

$$
\begin{aligned}
& \frac{A_{0}}{a^{2}}+\frac{p}{a^{2}} B_{0}+A_{1}+(p+2) B_{1}+\frac{\lambda \beta}{2 \mu a^{2}}+k h=0, \\
& \frac{A_{0}}{b^{2}}+\frac{p+2}{b^{2}} B_{0}-A_{1}-(p+2) B_{1}-\frac{\lambda \beta}{2 \mu b^{2}}=0, \\
& \left(\frac{1}{a^{2}}+\frac{3}{b^{2}}\right) A_{1}+\left(\frac{p}{a^{2}}+\frac{3 p+6}{b^{2}}\right) B_{1}+\left(\frac{1}{a^{2}}+\frac{3}{b^{2}}\right) k h=0, \\
& \left(\frac{3}{a^{2}}+\frac{1}{b^{2}}\right) A_{1}+\left(\frac{3 p+6}{a^{2}}+\frac{p+4}{b^{2}}\right) B_{1}=0, \\
& (1-p) B_{0}+\frac{\left(a^{2}-b^{2}\right)}{4}\left[(1-p) B_{1}+k h\right]-\frac{\lambda+2 \mu}{2 \mu} \beta=0 .
\end{aligned}
$$

As a numerical example, we shall take the dimensions of the section as $a=2 \mathrm{~cm}$, $b=1 \mathrm{~cm}$ and shall assume the values of the elastic constants as $\lambda=8.66 \times 10,{ }^{5} \mu=$ $8.20 \times 10^{5}$ (unit $\mathrm{Kg}$ weight per $\mathrm{cm}^{2}$ ). Substituting these values into (22) and solving the simultaneous equations, we find (unit $k \alpha$ )

$$
\begin{aligned}
A_{0} & =-0.5786, \quad A_{1}=-1.6793, \quad B_{0}=0.1156, \quad B_{1}=0.4627, \\
\beta & =0.4500 .
\end{aligned}
$$

In our calculation, the constant $\beta$ does not vanish. Hence we see that when a spiral rod is twisted, an axial elongation (or contraction) takes place. Substituting these numerical values of constants into Eq. (21), we calculate the stresses on the $x^{\prime}$ and $y^{\prime}$ axes, which are plotted in Figs. 1 and 2. In this case, the predominating stress which is concerned in torque is $X_{z}^{\prime}$ and its maximum amount is $1.6 \mu \alpha$. While the predominating stress among the other stresses is the normal stress $Z_{z}$, which attains its maximum amount of $5.44 k \mu \alpha$ at the points $x^{\prime}= \pm 2, y^{\prime}=0$, as is shown in the figures. Accordingly, 
the latter can not be ignored compared with the former except in cases when $k$ is extremely small.

The shape of the cross section is not deformed by twist in a straight rod, but in the

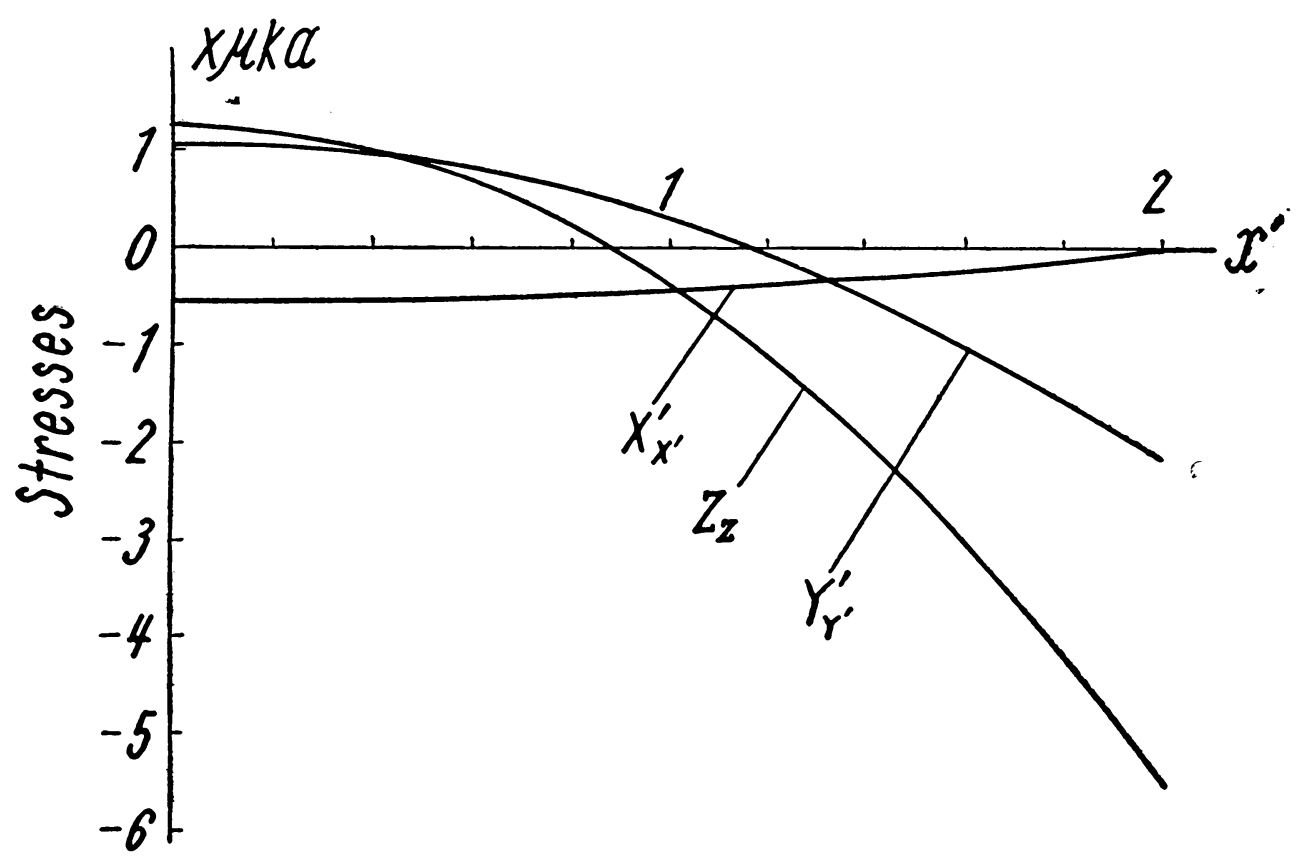

Fig. 1. Stresses on $x^{\prime}$ axis.

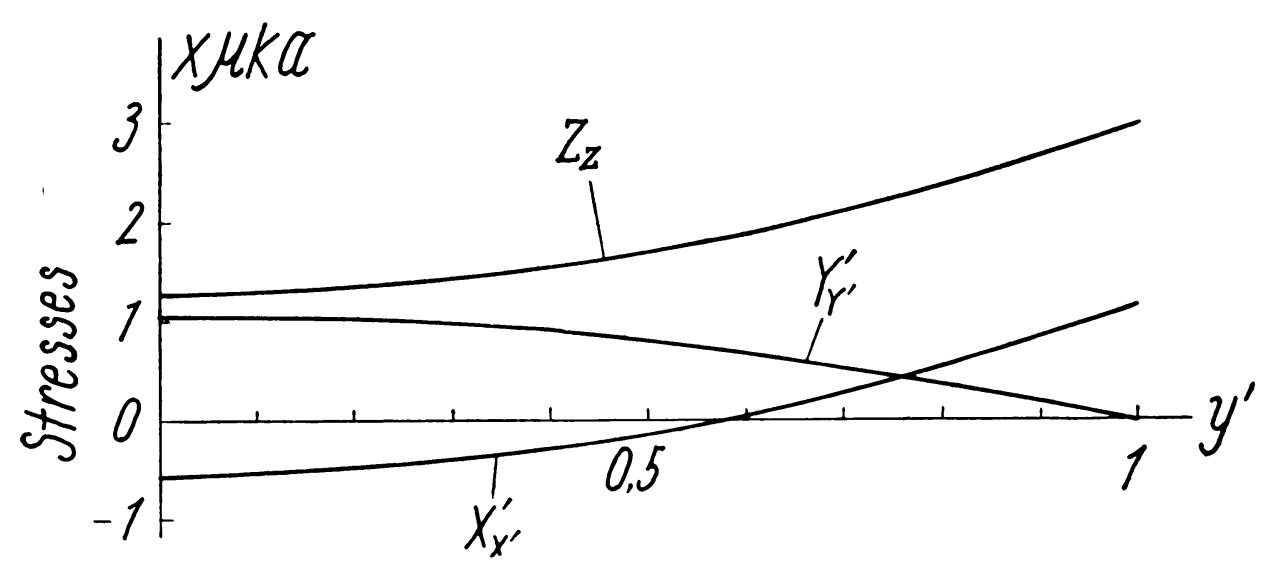

Fig. 2. Stresses on $y^{\prime}$ axis.

case of a spiral rod the distortion of the section, as shown in Fig. 3, is caused by twist.

Next, we shall treat the stretching problem. For convenience of calculations, let us put the displacements in the forms 


$$
\begin{aligned}
& u^{\prime}=u_{1}\left(x^{\prime}, y^{\prime}\right)-\gamma x^{\prime}-\alpha y^{\prime} z, \\
& v^{\prime}=v_{1}\left(x^{\prime}, y^{\prime}\right)-\gamma y^{\prime}+\alpha x^{\prime} z, \\
& w=w_{1}\left(x^{\prime}, y^{\prime}\right)+\beta z
\end{aligned}
$$

where

$$
\gamma=\frac{1}{2}(1-p) \beta .
$$

When $k$ is small, neglecting the smaller quantities of the order of $k^{3}$, the further calculations become quite similar to those of the previous case, viz., Eq. (11) is equally

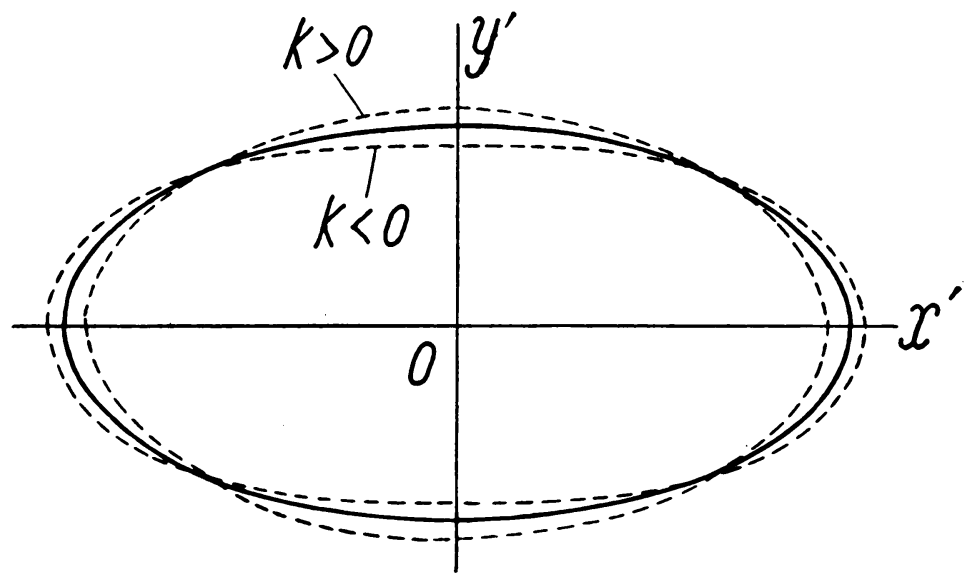

Fig. 3. Distortion of the section.

valid for this case and the stresses are represented in the same forms as in Eq. (18), except the following stresses

$$
\begin{aligned}
& X_{x^{\prime}}^{\prime}=2 \mu\left\{f_{1}^{\prime}+\overline{f_{1}^{\prime}}+p\left(f_{2}+\overline{f_{2}}\right)+x^{\prime}\left(f_{2}^{\prime}+\overline{f_{2}^{\prime}}\right)+k\left(\zeta f_{3}^{\prime}+\bar{\zeta} \overline{f_{3}^{\prime}}\right)\right\}, \\
& Y_{y^{\prime}}^{\prime}=-2 \mu\left\{f_{1}^{\prime}+\overline{f_{1}^{\prime}}+(p+2)\left(f_{2}+\overline{f_{2}}\right)+x^{\prime}\left(f_{2}^{\prime}+\overline{f_{2}^{\prime}}\right)\right\}, \\
& Z_{z}=-2 \mu\left\{(1-p)\left(f_{2}+\overline{f_{2}}\right)+k\left(\zeta f_{3}^{\prime}+\overline{\zeta f_{3}^{\prime}}\right)-\frac{1}{2}(3-p) \beta\right\} .
\end{aligned}
$$

For an elliptic section, we shall put

$$
2 f_{1}^{\prime}=A_{0}+A_{1} \zeta^{2}, \quad 2 f_{2}=B_{0}+B_{1} \zeta^{2}, \quad 2 f_{3}^{\prime}=C_{0} \zeta .
$$

The corresponding stresses become

$$
\begin{aligned}
& X_{x^{\prime}}^{\prime}=2 \mu\left\{A_{0}+p B_{0}+2 B_{1} x^{\prime 2}+\left(A_{1}+p B_{1}+k C_{0}\right)\left(x^{\prime 2}-y^{\prime 2}\right)\right\}, \\
& Y_{\nu^{\prime}}^{\prime}=-2 \mu\left\{A_{0}+(p+2) B_{0}+2 B_{1} x^{\prime 2}+\left[A_{1}+(p+2) B_{1}\right]\left(x^{\prime 2}-y^{\prime 2}\right)\right\}, \\
& Z_{z}=-2 \mu\left\{(1-p) B_{0}+\left[(1-p) B_{1}+k C_{0}\right]\left(x^{\prime 2}-y^{\prime 2}\right)-\frac{1}{2}(3-p) \beta\right\},
\end{aligned}
$$




$$
\begin{aligned}
& X_{y^{\prime}}^{\prime}=-2 \mu\left\{2 A_{1}+2(p+2) B_{1}+k C_{0}\right\} x^{\prime} y^{\prime}, \\
& X_{z}^{\prime}=-\mu\left(C_{0}+\alpha\right) y^{\prime}, \\
& Y_{z}^{\prime}=-\mu\left(C_{0}-\alpha\right) x^{\prime} .
\end{aligned}
$$

From the boundary condition (9) with the condition that the moment of couple due to the shearing stresses vanishes, we have a system of simultaneous equations for the determination of the unknown constants $A_{0}, A_{1}, B_{0}, B_{1}, C_{0}$ and $\alpha$, as

$$
\begin{gathered}
\left(a^{2}-b^{2}\right) C_{0}=\left(a^{2}+b^{2}\right) \alpha \\
\left(a^{2}+b^{2}\right) C_{0}=\left(a^{2}-b^{2}\right)\{\alpha+(3-p) k \beta\} \\
\frac{A_{0}}{a^{2}}+\frac{p}{a^{2}} B_{0}+A_{1}+(p+2) B_{1}+k C_{0}=0 \\
\left(\frac{1}{a^{2}}+\frac{3}{b^{2}}\right) A_{1}+\left(\frac{p}{a^{2}}+\frac{3 p+6}{b^{2}}\right) B_{1}+\frac{k C_{0}}{2}\left(\frac{1}{a^{2}}+\frac{5}{b^{2}}\right)+\frac{k \alpha}{2}\left(\frac{1}{b^{2}}-\frac{1}{a^{2}}\right)=0 \\
\frac{A_{0}}{b^{2}}+\frac{p+2}{b^{2}} B_{0}-A_{1}-(p+2) B_{1}=0 \\
\left(\frac{3}{a^{2}}+\frac{1}{b^{2}}\right) A_{1}+\left(\frac{3 p+6}{a^{2}}+\frac{p+4}{b^{2}}\right) B_{1}+\frac{k C_{0}}{2}\left(\frac{1}{a^{2}}+\frac{1}{b^{2}}\right)-\frac{k \alpha}{2}\left(\frac{1}{b^{2}}-\frac{1}{a^{2}}\right)=0 .
\end{gathered}
$$

If $q$ is the mean value of the axial normal stress over the section, then

$$
\begin{aligned}
q & =\frac{1}{\pi a b} \iint Z_{z} d x^{\prime} d y^{\prime} \\
& =\frac{E}{3-p}\left\{(3-p) \beta-2(1-p) B_{0}-\frac{1}{2}\left(a^{2}-b^{2}\right)\left[(1-p) B_{1}+k C_{0}\right]\right\},
\end{aligned}
$$

where $E$ is Young's modulus. From Eq. (28), $\beta$ can be expressed as a multiple of $q$.

We shall consider an elliptic section of the same dimensions as in the previous example. From Eqs. (27) and (28) we have

$$
\begin{array}{cl}
C_{0}=2.3565 k \beta, \quad \alpha=1.4139 k \beta, & A_{0}=-1.8851 k^{2} \beta, \\
B_{0}=0, \quad A_{1}=-4.8148 k^{2} \beta, & B_{1}=1.1783 k^{2} \beta,
\end{array}
$$

and

$$
\beta=\frac{q}{E\left\{1-1.7673 k^{2}\right\}} .
$$

Accordingly a twist arises when a spiral rod is pulled axially, since $\alpha$ does not vanish in our calculations. The normal stresses $X_{x^{\prime}}^{\prime}, Y_{y^{\prime}}^{\prime}$ attain their maximum value of 1.5 $k^{2} E \beta$ at the center of the section, and so they are very small quantities compared with $Z_{z}$. The normal stress $Z_{z}$ is distributed almost uniformly over the section and it attains 
its maximum and minimum values of $\left(1+2.356 k^{2}\right) E \beta$ and $\left(1-9.425 k^{2}\right) E \beta$ at both ends of the minor and major axes of the ellipse respectively. The predominating stress among the shearing stresses is $X_{z}^{\prime}$ and it attains its maximum value of $1.5 k E \beta$ at both ends of the minor axis.

In conclusion, the writer wishes to express his thanks to Miss E. Itagaki, his assistant, for her earnest help in this study. 